\title{
Collective and Tracer Diffusion via a Defect Cluster in LSGM
}

\author{
I.V. Belova ${ }^{1, a}$, G.E. Murch ${ }^{1, b}$, D. Samuelis ${ }^{2, c}$ and M. Martin ${ }^{2, d}$ \\ ${ }^{1}$ Diffusion in Solids Group, School of Engineering, \\ The University of Newcastle, Callaghan, New South Wales 2308, Australia \\ ${ }^{2}$ Institute of Physical Chemistry, RWTH Aachen University, Landoltweg 2, 52056 Aachen Germany

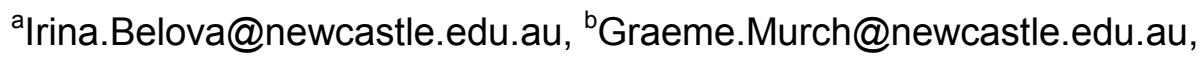 \\ 'samuelis@pc.rwth-aachen.de, ${ }^{\mathrm{d}}$ martin@rwth-aachen.de
}

Keywords: Diffusion, LSGM, vacancies, cluster mechanism.

\begin{abstract}
It was recently shown by Schulz and co-workers that in doped lanthanum gallate: $\mathrm{La}_{(1-\mathrm{x})}$ $\mathrm{Sr}_{\mathrm{x}} \mathrm{Ga}_{(1-\mathrm{y})} \mathrm{Mg}_{\mathrm{y}} \mathrm{O}_{(3-(\mathrm{x}+\mathrm{y}) / 2)}$ (LSGM) the three cations: $\mathrm{La}, \mathrm{Sr}$ and $\mathrm{Mg}$ have tracer diffusivities that are nearly identical. To explain these findings, a bound defect cluster mechanism containing a cation vacancy from both the A- and the B- sublattices and an anion vacancy was proposed as the principal vehicle for cation diffusion in LSGM. In this paper, implications of this mechanism are considered for the first time. Sum-rule expressions for the collective correlation factors are derived and found to be in excellent agreement with Monte Carlo calculations. Expressions are also developed for the tracer correlation factors of lanthanum and gallium for diffusion via the cluster mechanism and tested by Monte Carlo computer simulation. Good agreement was found. The ratio of the tracer diffusivities of lanthanum and gallium are shown to be consistent with the cluster mechanism.
\end{abstract}

\section{Introduction}

Recently it was shown by Schulz et al. [1,2] that in doped lanthanum gallate: $L a_{(1-x)} S r_{x} G a_{(1-y)} M g_{y}$ $O_{(3-(x+y) / 2)}$ or LSGM, the three cations investigated: $\mathrm{La}, \mathrm{Sr}$ and $\mathrm{Mg}$ all have tracer diffusion coefficients that are nearly identical ${ }^{1}$. Importantly, a similar behavior was also found for the impurities $\mathrm{Y}, \mathrm{Nd}$ and $\mathrm{Fe}$ [3]. To explain these experimental findings a bound defect cluster mechanism containing cation vacancies of both the $A$ - and the $B$-sublattice and anion vacancies was proposed as the principal vehicle for cation diffusion in LSGM [2]. Three different cluster mechanisms are possible in the lanthanum gallate structure. They consist of two cation vacancies (different sublattices) and one, two or three anion vacancies. In each mechanism the cations of a given type do not occupy the other cation sublattice. As was pointed out in [2] only a one anion vacancy cluster can produce long range diffusion (the others would require partial dissociation of the anion vacancies).

On the other hand, the anion vacancies are many orders of magnitude more mobile than the cation vacancies in this structure. This makes it possible for the cluster mechanism to be very dynamic on the oxygen sublattice in the sense that oxygen vacancies only 'visit' clusters from time to time: they are not really permanently bound to the clusters. Once an oxygen vacancy is available in the required location for the cluster itself to be able to move, a few jumps can then occur on the cation sublattices. These jumps seemed to proceed only in a highly correlated way making the two cation vacancies tightly bound. These considerations lead to the necessity of analyzing first the triple defect (two cation vacancies and one anion vacancy) cluster mechanism and then the cation vacancy-pair with a supporting role of the anion vacancies. With the addition of doping elements on both cation sublattices the total number of anion vacancies increases greatly. This makes the model

\footnotetext{
${ }^{1}$ It was not possible to measure the $G a$ tracer diffusion coefficient, owing to the natural abundances of the two stable gallium isotopes, ${ }^{69} \mathrm{Ga}$ and ${ }^{71} \mathrm{Ga}$, being $60 \%$ and $40 \%$ respectively. The maximum enrichment of, say, ${ }^{71} \mathrm{Ga}$ above this background level is therefore a factor of 2.5 , which is insufficient for determining diffusion profiles.
} 
of the cation vacancy-pair mechanism with support by the anion vacancies even more relevant to the case of diffusion in LSGM.

The absence of any information about tracer (and collective) correlation effects associated with the cluster mechanism makes any conclusions about their role in diffusion almost impossible. In the present paper we initiate an investigation of the implications of the cluster mechanism on such correlations in cation diffusion in un-doped lanthanum gallate.

\section{Theoretical Models for Un-doped Lanthanum Gallate}

I. Collective Diffusion. The cluster mechanism first proposed in [2] can be considered in the following way for the undoped oxide $\mathrm{ABO}_{3}$. First, we define possible vector-jumps of the $A$ cation (lanthanum) vacancy in the given cluster as $\vec{r}_{A}^{1}$ and $\vec{r}_{A}^{2}$, of the $B$ cation (gallium) vacancy as $\vec{r}_{B}$, and the $O$ vacancy as $\vec{r}_{O}^{1}$ and $\vec{r}_{O}^{2}$ (see Fig. 1). From geometrical considerations we have that:

$$
\vec{r}_{A}^{1}+\vec{r}_{A}^{2}+2\left(\vec{r}_{O}^{1}+\vec{r}_{O}^{2}\right)+2 \vec{r}_{B}=\overrightarrow{0}
$$

and

$$
\left|\vec{r}_{A}^{i}\right|=\left|\vec{r}_{B}\right|=\sqrt{2}\left|\vec{r}_{O}^{i}\right|=a
$$

where $a$ is the lattice parameter. We can now introduce phenomenological coefficients $L_{A A}, L_{B B}$ and $L_{O O}$ and their correlated parts, the collective correlation factors $f_{i j}^{(j)}$ for the diffusion of cations $A$ and $B$ and oxygen by way of the postulated cluster diffusion mechanism:

$$
L_{i j}=f_{i j}^{(j)} L_{j j}^{(0)},
$$

where the superscript (0) always indicates an uncorrelated part.
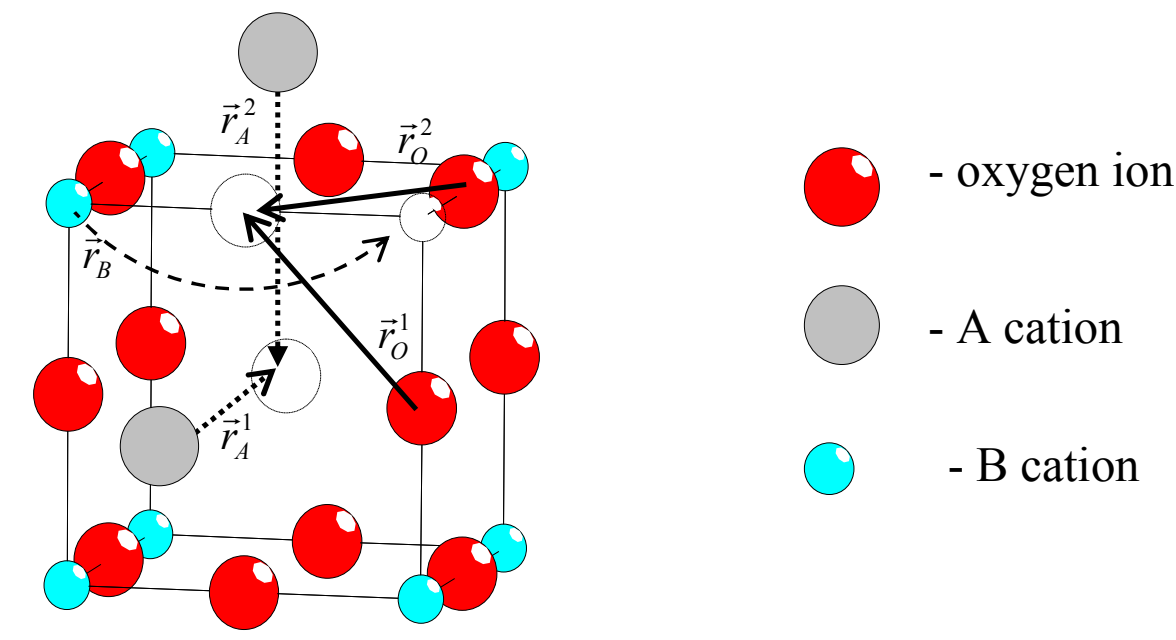

Fig. 1 Schematic representation of the possible jumps of the vacancies in the cluster in the lanthanum gallate structure. Unfilled circles represent vacancies.

The Einsteinian expression for the phenomenological coefficients is [4]:

$$
L_{i j}=\frac{<R_{i} \cdot R_{j}>}{6 V k T},
$$


where $R_{i}$ is the collective displacement of species $i$ in the volume $V$ in time $t$ and $k$ and $T$ are Boltzmann constant and temperature respectively. It is a natural requirement of the vacancy-cluster mechanism that the cation and anion vacancies have the same displacement after some long time $t$. Accordingly, the collective displacements of the cations and anions must therefore be equal, i.e. $R_{A}$ $=R_{B}=R_{O}$. We then have that:

$L_{A A}=L_{B B}=L_{O O}=L_{A O}=L_{B O}=L_{A B}$

Using exactly the same approach as given by Moleko and Allnatt [5] see also Allnatt and Lidiard [6] and Eq. 1, we soon have the following Sum-rule relations relating the phenomenological coefficients:

$$
\begin{aligned}
& 2 L_{O O}+\frac{w_{O}}{w_{A}} L_{A O}+2 \frac{w_{O}}{w_{B}} L_{B O}=2 L_{O O}^{(0)}, \\
& 2 L_{B B}+2 \frac{w_{B}}{w_{O}} L_{B O}+\frac{w_{B}}{w_{A}} L_{A B}=2 L_{B B}^{(0)},
\end{aligned}
$$

where $w_{O}$ is the exchange frequency of a vacancy (of the vacancy-cluster) with an oxygen ion and $w_{A}$ and $w_{B}$ are the exchange frequencies of the cation vacancies (of the vacancy-cluster).

The expressions for the uncorrelated parts $L_{\mathrm{ii}}{ }^{(0)}$ of the phenomenological coefficients can be found by inspection as:

$$
L_{i i}^{(0)}=\frac{z_{i} r_{i}^{2} c_{i} w_{i} c_{V i} N_{i}}{6 V k T}
$$

where the 'jump' coordination numbers are $z_{O}=2, z_{A}=2, z_{B}=1$, the oxygen ion composition $c_{O}=1$ and the cation composition $c_{A}=1, c_{B}=1$ with one cluster and therefore $c_{V i} N_{i}=1$ where $c_{V i}$ is the vacancy composition as 'seen' by component $i$ and $N_{i}$ is the number of sites on the corresponding sublattice ( $i=A, B, O)$. We then have the Sum-rule relations Eq. 6 in terms of the correlated parts (the collective correlation factors) of the phenomenological coefficients:

$$
\begin{aligned}
& f_{A A}=f_{A O}^{(A)}=f_{A B}^{(A)}=\frac{1}{1+2 w_{A} / w_{O}+2 w_{A} / w_{B}}, f_{B B}=f_{B O}^{(B)}=f_{A B}^{(B)}=\frac{2}{2+2 w_{B} / w_{O}+w_{B} / w_{A}}, \\
& f_{O O}=f_{A O}^{(O)}=f_{B O}^{(O)}=\frac{2}{2+w_{O} / w_{A}+2 w_{O} / w_{B}} .
\end{aligned}
$$

Using standard procedures [7,8] we performed Monte Carlo calculations of the collective correlation factors $f_{A A}, f_{B B}$ and $f_{A B}{ }^{\left({ }^{(}\right)}$and $f_{A B}{ }^{(B)}$ as functions of the ratio of exchange frequencies $w_{A} / w_{O}$ and $w_{B} / w_{O}$. The cell size for the calculations was $40 \times 40 \times 40$, the number of jumps per atom was 10 and the number of observations was 2500 . We made use of the Einsteinian expression for the collective correlation factors:

$$
f_{i j}^{(i)}=\frac{<R_{i} \cdot R_{j}>}{6 V k t L_{i i}^{(0)}}=\frac{<R_{i} \cdot R_{j}>}{n_{i} r_{i}^{2} / N_{i}},
$$

where $R_{i}$ is the total displacement of all the atoms of type $i, n_{i}=z_{i} c_{i} w_{i} N_{i}$-total number of jumps of atoms of the type $i$ in time $t$. 


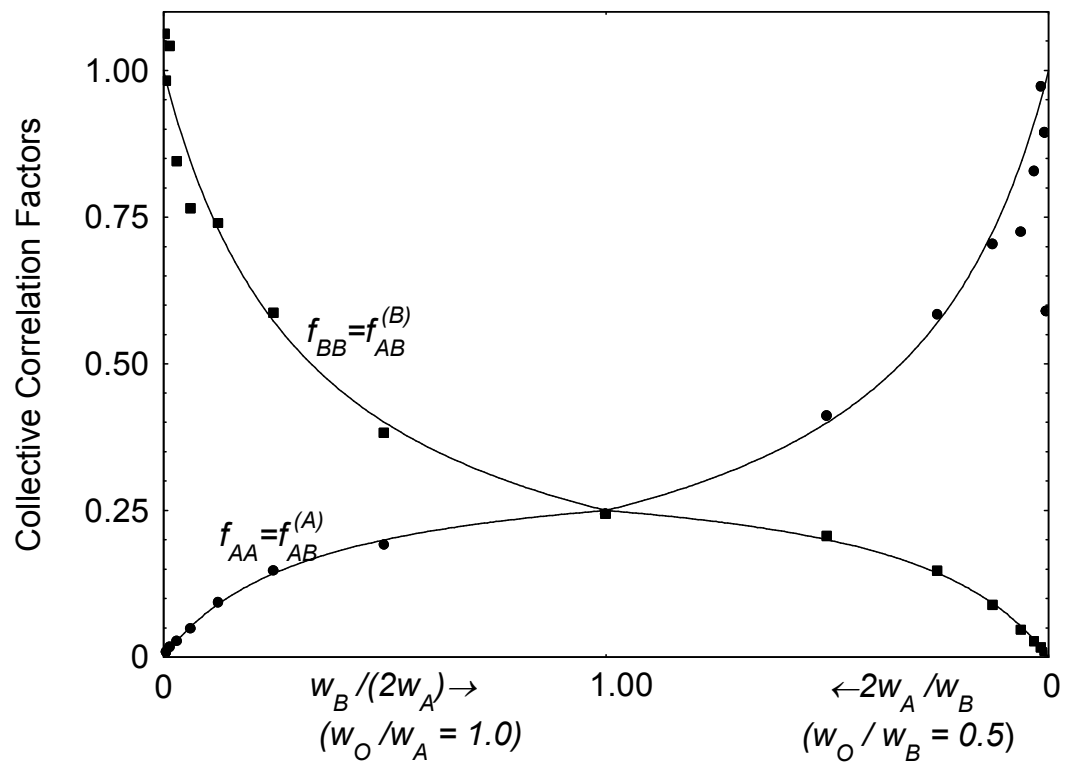

Fig. 2 Collective correlation factors as functions of the ratios of the exchange frequencies. Symbols - Monte Carlo simulation results, lines - analytical relations derived on the basis of the Sum-rule.
It is seen that there is perfect agreement between the Monte Carlo computer simulation results (presented as symbols in Fig. 2) and the theoretical expressions (solid lines in the same figure).

These relationships between the phenomenological coefficients will be extended elsewhere for the doped oxide with the hope of suggesting critical interdiffusion and/or demixing experiments to test the existence of the cluster mechanism.

II. Tracer Diffusion. In this section, we develop expressions for the tracer correlation factors for cations and anions $\left(f_{A}, f_{B}\right.$ and $\left.f_{O}\right)$ along similar lines to that described by Belova and Murch [9]. First, we note that the diagonal collective correlation factors are given as (Eq. 8):

$$
f_{V A}=4 f_{A A} ; \quad f_{V B}=4 f_{B B} ; \quad f_{V O}=4 f_{O O},
$$

where $f_{V A}, f_{V B}$ and $f_{V O}$ are the correlation factors of the vacancy-cluster ends. We accept the point, where $w_{B}=2 w_{A}$ as the reference point for determining the geometric tracer correlation factors $f_{O(A)}$ and $f_{O(B)}$ and the corresponding quantities $M_{O(A)}=2 f_{O(A)} /\left(1-f_{O(A)}\right)$ and $M_{O(B)}=2 f_{O(B)} /\left(1-f_{O(B)}\right)$. From Monte Carlo simulations (see below) we have that $f_{0(A)}=0.413$ and $f_{0(B)}=0.212$ with the value for $M_{0(A)}=1.407$ and, similarly, $M_{0(B)}=0.538$. Then, using Manning's formalism [9] for the tracer correlation factors, we can write:

$$
f_{A}=\frac{H_{A}}{2 w_{A}+H_{A}}, \quad f_{B}=\frac{H_{B}}{2 w_{B}+H_{B}}
$$

where $H_{i}=f_{V i} W_{i} M_{0(i)}$ :

$$
H_{A}=\frac{4 M_{0(A)} w_{A} w_{B} w_{O}}{2 w_{A} w_{O}+w_{B} w_{O}+2 w_{A} w_{B}}, H_{B}=\frac{8 M_{0(B)} w_{A} w_{B} w_{O}}{2 w_{A} w_{O}+w_{B} w_{O}+2 w_{A} w_{B}} .
$$

Eq. 12, together with the expressions Eq. 11, gives the final set of expressions for the tracer correlation factors $f_{A}$ and $f_{B}$.

In order to calculate by Monte Carlo simulation the tracer (or self) correlation factors we use similar Einsteinian expression as Eq. 9 but now in terms of the individual displacement $r_{j}$ of a tracer atom of type $j$ and the corresponding average number of jumps of the atom $m_{j}$ in time $t[7,11]$ :

$$
f_{j}=\frac{<r_{j}^{2}>}{m_{j} r_{j}^{2}} \text {. }
$$


In Fig. 3 we present results of Monte Carlo simulations for tracer (self) correlation factors over the full range of the ratio $w_{A} / w_{B}$ with specified $w_{O}$. The agreement between computer simulation results and the theoretical approach is generally good. Differences can be attributed to the wellknown shortcomings of the Manning formalism in describing tracer diffusion [6]. The likely usual experimental condition is one where the oxygen ion exchange frequency with a vacancy at one end of the vacancy-cluster is very large compared with the cation frequencies with the vacancies at the other ends. It is seen that the tracer correlation factor for the cation then extrapolates to 0.654 the value for diffusion by isolated vacancies in the s.c. lattice. The corresponding tracer correlation factor for the oxygen ions then extrapolates to zero. This comes about because the very slow moving ends of the vacancy-cluster mean that most jumps of the fast moving vacancy at the oxygen ion end will effectively be reversed. This directly affects the tracer correlation factor of the oxygen ions that jump with such vacancies of the vacancy-cluster. Of course in real (doped) material the fraction of oxygen vacancies actually moving in this way is extremely small compared with the very high concentration of isolated oxygen ion vacancies and accordingly there would be no discernible effect on the overall oxygen ion diffusivity.

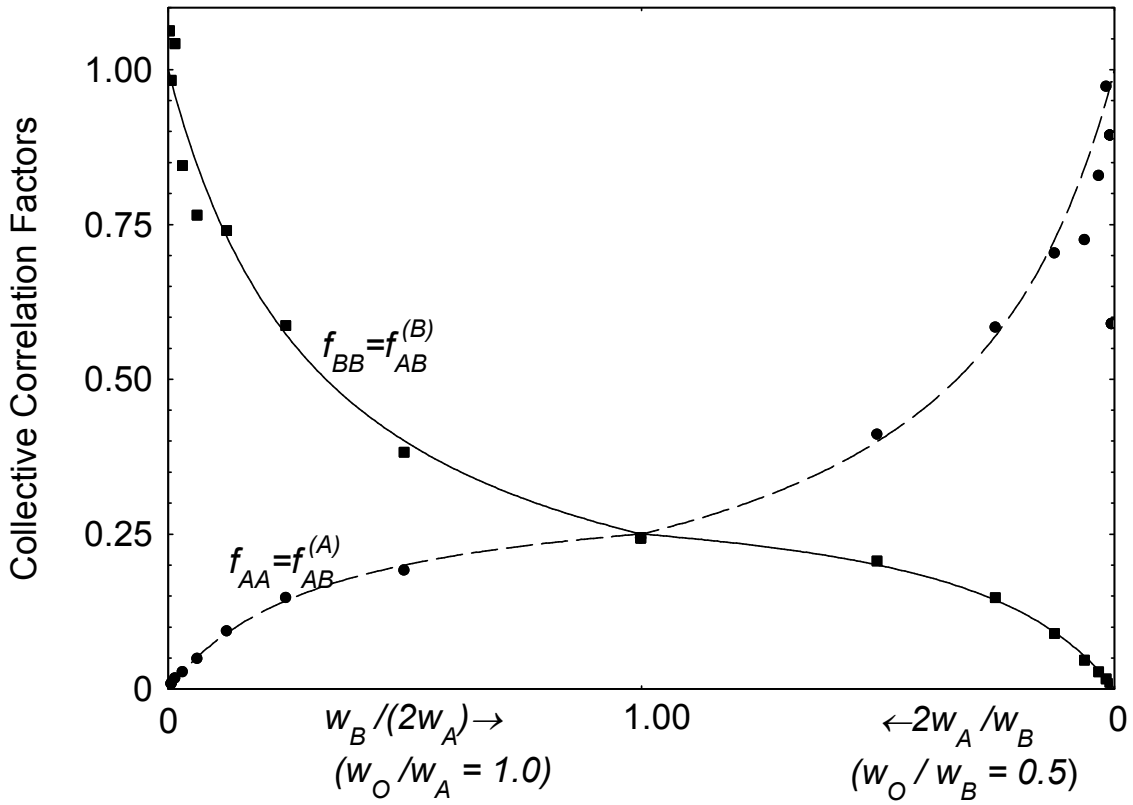

Fig. 3 Tracer correlation factors as functions of the ratios of the exchange frequencies of the vacancy ends in the cluster mechanism. Symbols: Monte Carlo simulation results; solid and dashed lines: Manning type of analytical approximation (Eqns. 10,11).
In Fig. 4 we present results of calculations of the ratio of the tracer diffusion coefficients $D_{A}{ }^{*} / D_{B}{ }^{*}$ as functions of the ratios of the exchange frequencies. It is seen that the maximum of this ratio is slightly under fourteen (and this follows the behavior of the vacancy-pair mechanism in the $B 2$ structure), whereas the maximum of $D_{B}{ }^{*} / D_{A}{ }^{*}$ is likely to be well under ten.

On the other hand, from analysis of Fig. 4 we can see that the limiting values for the ratio of the cation tracer diffusivities are developed at rather high ratios of the (cation) exchange frequencies when the oxygen vacancy moves faster than the other vacancies in the cluster. Preliminary results for the $w_{O} / w_{B}=1000$ case (not shown) also seem to sit about where the $w_{O} / w_{B}=100$ results sit, strongly suggesting that the latter curve is already about the limiting one. Let us consider restricted intervals of, say, $[0.1,1]$ on the left hand side and $[1,0.1]$ on the right hand side of the abscissa of Fig. 3. These restricted intervals are likely to encompass the ratios of exchange frequencies encountered in practice. We then have the limits of about 2 and 1/5 for the ratio of the cation diffusivities. This 'window' is consistent with experimentally obtained values for the ratio of the various cation diffusivities (circa unity) for the doped oxide [2,3]. When the doped oxide is considered we should take into account that for the composition of 0.1 of the additional elements on each cation sublattice the resulting (or average) cation vacancy exchange frequency will be almost unaffected by the (possible) different mobility of the added element. This will mean that the general limits of the ratios of the tracer diffusivities even in the doped oxide can be expected to follow the behavior in the un-doped oxide considered here. 


\section{Summary}

In this paper, implications of a postulated cluster mechanism in LSGM mechanism are considered for the first time. Sum-rule expressions for the collective correlation factors are derived and found to be in excellent agreement with Monte Carlo calculations. Expressions are also developed for the tracer correlation factors of lanthanum and gallium for diffusion via the cluster mechanism and tested by Monte Carlo computer simulation. Good agreement was found. The ratio of the tracer diffusivities of lanthanum and gallium are shown to be consistent with the cluster mechanism.

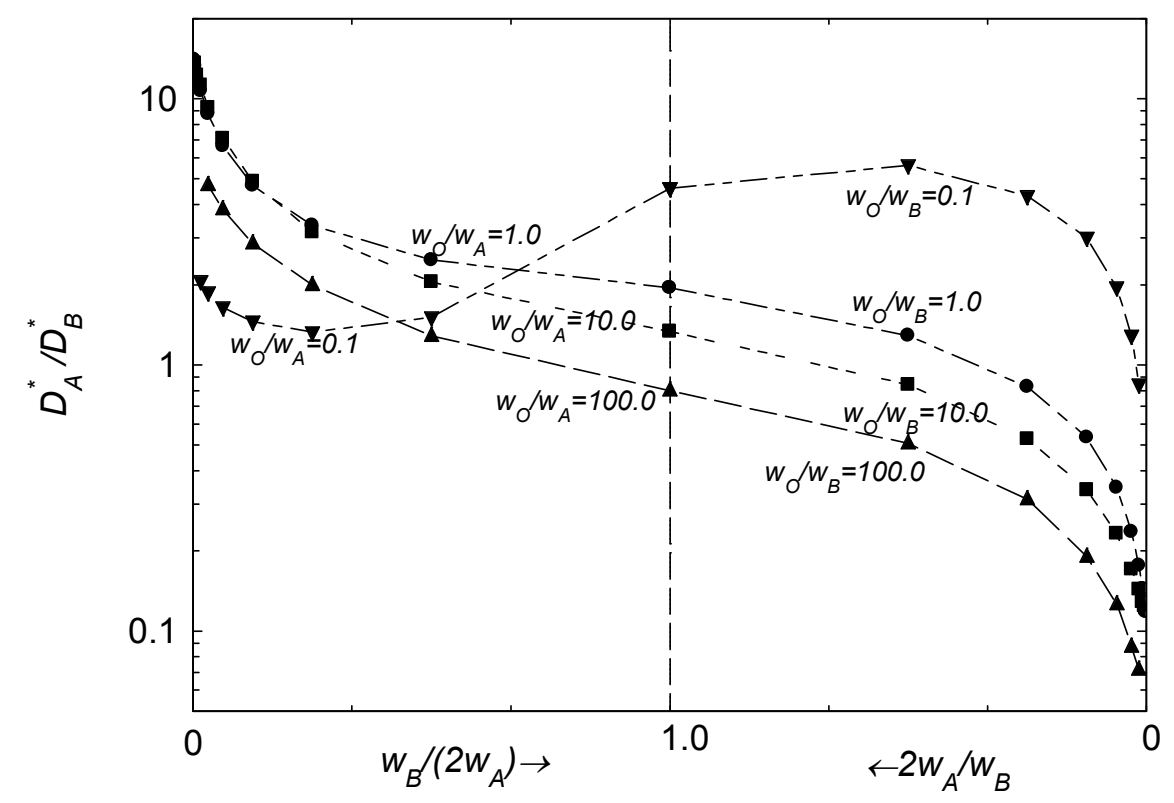

Fig. 4 Ratio of the cation tracer diffusivities as a function of the ratios of the exchange frequencies as calculated by Monte Carlo simulations.

\section{Acknowledgements}

We wish to thank the Australian Research Council for its support of this work under the Discovery Project Grants Scheme.

\section{References}

[1] O. Schulz and M. Martin: Solid State Ionics, Vol. 135 (2000), p. 549

[2] O. Schulz, M. Martin, Ch. Argirusis and G. Borchardt: Phys. Chem. Chem. Phys. Vol. 5 (2003), p. 2308

[3] O. Schulz, S. Flege and M. Martin, Solid Oxide Fuel Cells VIII (SOFC-VIII), Eds. S.C. Singhal and M. Dokiya, Proceedings of the Electrochemical Society, PV 2003-2007 (2003) p. 304

[4] A.R. Allnatt: J. Phys. C: Solid St. Phys. Vol. 15 (1982), p. 5605

[5] L.K. Moleko and A.R. Allnatt: Phil. Mag. A 58 (1988), p. 677

[6] A.R. Allnatt and A.B. Lidiard: Atomic Transport in Solids (Cambridge University Press, Cambridge 1993).

[7] A.R. Allnatt and E.L. Allnatt: Phil. Mag. A Vol. 49 (1984), p. 625.

[8] G.E. Murch: Diffusion in Crystalline Solids, edited by G.E. Murch and A.S. Nowick, Academic press, Orlando Fl (1984), p. 379

[9] I.V. Belova and G.E. Murch: Phil. Mag. A Vol. 79 (1999), p. 1509

[10] J.R. Manning: Phys. Rev. B, Vol. 4 (1971) p. 1111

[11]H.J. DeBruin and G.E. Murch: Phil. Mag. Vol. 27 (1973), p. 1475 
Diffusion and Thermodynamics of Materials

doi:10.4028/3-908451-35-3

Collective and Tracer Diffusion via a Defect Cluster in LSGM

doi:10.4028/3-908451-35-3.81 